\title{
BARGAINING AND COOPERATION IN STRATEGIC FORM GAMES
}

\author{
Sergiu Hart \\ Hebrew University of Jerusalem
}

\author{
Andreu Mas-Colell \\ Universitat Pompeu Fabra \\ Barcelona Graduate School \\ of Economics
}

\begin{abstract}
In this paper we view bargaining and cooperation as an interaction superimposed on a game in strategic form. A multistage bargaining procedure for $N$ players, the "proposer commitment" procedure, is presented. It is inspired by Nash's two-player variable-threat model; a key feature is the commitment to "threats." We establish links to classical cooperative game theory solutions, such as the Shapley value in the transferable utility case. However, we show that even in standard pure exchange economies, the traditional coalitional function may not be adequate when utilities are not transferable. (JEL: C70, C71, C78, D70)
\end{abstract}

\section{Introduction}

The general equilibrium approach to economic modeling has familiarized us with the idea that the construction of a good theory should be grounded in a sharp conceptual distinction between the underlying data of the economy (e.g., preferences, endowments, and technologies), to be thought of as fixed, and the institutions that operate upon them (e.g., markets), which are more variable and subject to design (with the aim, most frequently, of inducing cooperation and efficient outcomes). In this paper we focus on bargaining theory and take the position that general bargaining theory should be viewed in a similar way. Specifically:

(i) There is an underlying physical reality represented by a game in strategic form; that is, there is a number of players $N$, and, for each one of them, a set

\footnotetext{
The editors in charge of this paper were Matthew O. Jackson and Xavier Vives.

Acknowledgments: A previous version (entitled "Cooperative Games in Strategic Form") was distributed in May 2008. The 2006 European Economic Association Presidential Address of the second author was partly based on this paper. The authors thank Robert Aumann, Elchanan BenPorath, Ken Binmore, Gil Kalai, Jean-François Mertens, Roger Myerson, Motty Perry, Andrew Postlewaite, Martin Shubik, Lloyd Shapley, Eyal Winter, Shmuel Zamir, the anonymous referees, and the editors for useful discussions and comments. Research partially supported by the Israel Science Foundation; the Fundación BBVA; the Ministerio de Educación y Ciencia, Spain, through a Consolider Grant; the Government of Catalonia, through the Barcelona GSE Research Network; and the Institute for Advanced Studies at the Hebrew University of Jerusalem.
}

E-mail addresses: Hart: hart@ huji.ac.il; Mas-Colell: andreu.mascolell@gmail.com 
of actions and a payoff function that associates a payoff (or utility) to each combination of actions played by the different players.

(ii) Bargaining is a non-cooperative interaction that acts through an institutional setup superimposed on the strategic form. In particular, the institutional setup provides the players with the possibility of reaching binding agreements. We refer to the whole as a "bargaining procedure." It is the analog of the markets in the Walrasian general equilibrium theory. In a First Welfare Theorem fashion we could then ask, for example, if the "institution," namely, the bargaining procedure, induces bargaining processes that lead to a selection of final play that is (Pareto) efficient. Note that without an institution, and moreover one of a special sort, there is no reason for strategic interactions to lead to efficiency (recall for instance the prisoner's dilemma).

This differs from the existing approaches that first derive a cooperative game (i.e., a coalitional function) from the given strategic-form game, and then apply to it either a cooperative solution or a noncooperative bargaining procedure (there is a large literature here; see, for example, the surveys in the volume edited by Hart and Mas-Colell (1997)). One advantage of our proposed approach is that it sidesteps the difficulties that arise in defining the coalitional function, particularly in the non-transferable utility (NTU) case (more on this subsequently).

It is, of course, befitting to build a general theory of bargaining over a strategic form. In fact, it was already the position taken by Nash (1953) in his proposal for the endogeneization of the threat points of his two-person axiomatic theory of bargaining (Nash 1950), a proposal that was extended to $N$-person situations by Harsanyi $(1959,1963)$.

At a more specific level, our outlook is also inspired by the cooperative approach in game theory. As in cooperative game theory, we handle situations with an arbitrary number of players, and, more of the essence, we recognize that for the bargaining process to be sensible it is all-important to allow for the possibility of partial breakdown of the negotiation (in cooperative games, partial breakdown is captured by the fact that the "coalitional function" specifies what can be obtained by every coalition, thus contemplating the possibility of cooperation by subgroups smaller than the grand coalition). It is worth mentioning that in cooperative game theory the need for a strategic-form foundation has been persistently felt. One suggestion for filling the need was provided for the transferable utility ("TU") case by the founders (von Neumann and Morgenstern (1944) proposed to define what a coalition could reach as the maxmin level for the sum of the payoffs of the members of the coalition), and later generalized (Aumann 1959) to the non-transferable utility ("NTU") case in the guise of two coalitional forms (the "alpha" and the "beta"). Dissatisfaction with these definitions drew attention to particular classes of games where the determination of the coalitional form appeared uncontroversial (the "c-games" of Shapley-Shubik, a leading example 
of which are exchange economies; see Shubik (1983), Section 6.2.2). In such games, the theory of bargaining could be nicely factored through the coalitional form. We will have some opportunity to debate this point (see Section 7 below).

Having argued, up to this point, the importance of grounding matters in the strategic form we shall from now on proceed much more concretely by considering a special instance of a bargaining procedure, which we call the "proposer commitment" (PC) procedure. Although stylized, we believe that it captures some essential features of bargaining, in particular the fact that for participants in a bargaining process, putting proposals on the table is on the one hand a present opportunity (we assume that at any moment the current proposal has the monopoly of the floor), and on the other hand a risk of an opportunity lost (we assume that if a proposal is rejected then there is a chance that the proposer will never be able to make further proposals); clearly, these two considerations need to be balanced. We do not pretend that the PC procedure is the most general procedure because plainly it is not, but we submit that it is comparatively simple and that it captures some important features of bargaining, allowing one to pose some interesting questions and illuminating a number of significant phenomena.

The PC procedure has two sources of inspiration. The first one is the bargaining procedure formulated by ourselves (Hart and Mas-Colell 1996a) for the context where the underlying reality is a game in coalitional form. The sequential nature of the announcements and proposals we take from there, along with the idea that a rejected proposer becomes passive for the rest of the game (with some probability). But the consideration of a strategic form as the underlying reality allows - and in fact demands - that we enrich the determination of what happens to the play of a rejected proposer.

Second, the PC procedure is inspired by Nash (1953) in a crucial feature: We assume that players formulate and commit to threats (and that, therefore, commitment is possible). We view this commitment feature as going hand in hand with the set of strong institutions that must be in place if, as we assume, the outcome of bargaining is enforceable. We depart from Nash (1953) in an important respect, however: At each step of the negotiation only one player (the "proposer") makes threats. We do so because we want a bargaining procedure that, in the spirit of modern bargaining theory (see, e.g., Binmore, Osborne and Rubinstein 1992), has as players the players of the original, underlying strategic form. The twoplayer simultaneous threats model of Nash (1953) can be made to pass this test (replace, for example, the axiomatically based part of his solution by bargaining in the style of Stahl-Rubinstein; see, e.g., Osborne and Rubinstein (1990) and Houba and Bolt (2002)), but the $N$-player generalization of Harsanyi $(1959,1963)$ does not, at least to our knowledge (Harsanyi defines a sort of noncooperative bargaining, but it is between fictitious players, one for each coalition-and not between the original players of the game). Thus, in a sense (reminiscent of Shapley 
1969), we could also present our suggested solution as a simplification of the Nash-Harsanyi approach.

The paper is organized as follows. In Section 2 the basic model and the PC bargaining procedure are presented. In Section 3 we establish the standard existence and optimality properties. In Section 4 we focus on two special cases: two-person games and games with transferable utility. In Section 5 we discuss, in a particular context where the threats turn out to be "fixed" in equilibrium, a general connection of the PC solution with the Shapley value in the TU case. In Sections 6 and 7 we re-examine the concept of c-games (in particular, in the standard context of exchange economies) as candidates for the sort of simplification that would allow the factorization of bargaining analysis through a coalitional form (deducible from the fundamentals of the game). We discover an important difference between TU games-for which the simplification is possible (see Section 6) - and general NTU games - for which it is not. We show the latter in Section 7 by means of an example related to the transfer paradoxes of general equilibrium theory. Finally, Section 8 is devoted to a summary and a discussion of possible extensions and variations.

\section{The Model}

The basic data is an $N$-person game in strategic form $G=\left(N,\left(A^{i}\right)_{i \in N},\left(u^{i}\right)_{i \in N}\right)$, where $N$ is a finite set of players, and each player $i \in N$ has a finite set of actions $A^{i}$ and a payoff function $u^{i}: A \rightarrow \mathbb{R}$, with $A:=\prod_{i \in N} A^{i}$. A mixed action of player $i$ is $x^{i} \in \Delta\left(A^{i}\right)$, where $\Delta\left(A^{i}\right)=\left\{\left(x^{i}\left(a^{i}\right) \in \mathbb{R}_{+}^{A^{i}}: \sum_{a^{i} \in A^{i}} x^{i}\left(a^{i}\right)=1\right\}\right.$ is the probability simplex on $A^{i}$.

For each set of players $S \subset N$ (a coalition), let $A^{S}:=\prod_{i \in S} A^{i}$ denote the set of pure action combinations of the members of $S$. A correlated action of $S$ is $z^{S} \in$ $\Delta\left(A^{S}\right)$, a probability distribution on pure action combinations of $S$. The payoff functions are as usual multilinearly extended to mixed and correlated actions.

\subsection{The Proposer Commitment (PC) Procedure}

We now introduce the basic bargaining procedure.

Let $0 \leq \rho<1$ be a fixed parameter; think of $\rho$ as the probability of "repeat." The bargaining proceeds in rounds. In each round there is a set $S \subset N$ of "active" players, the actions of each "inactive" player $j \notin S$ being fixed at some $b^{j} \in A^{j}$; put $b^{N \backslash S}=\left(b^{j}\right)_{j \in N \backslash S}$. We will refer to $\omega=\left(S, b^{N \backslash S}\right)$ as a state. Initially, everyone is active, that is, $S=N$ (and so the starting state is $(N, \cdot)$ ). Each round, with state $\omega=\left(S, b^{N \backslash S}\right)$, proceeds as follows.

1. A "proposer" $k \in S$ is selected out of $S$ at random, with all members of $S$ being equally likely to be selected. 
2. The proposer $k$ chooses a pair $\left(z^{S}, x^{k}\right)$, where $z^{S} \in \Delta\left(A^{S}\right)$ is a correlated action of coalition $S$ and $x^{k} \in \Delta\left(A^{k}\right)$ is a mixed action of player $k$; think of $z^{S}$ as a "proposed agreement" for $S$, and of $x^{k}$ as a "threat."

3. Each player in $S$ is asked, in some order (deterministic or random), whether he accepts or rejects the proposed agreement $z^{S}$.

4. If all players in $S$ agree to $z^{S}$, then the procedure ends as follows: A joint action $a^{S} \in A^{S}$ is selected according to the distribution $z^{S}$, and the $N$-tuple of actions $\left(a^{S}, b^{N \backslash S}\right) \in A$ is played in the original strategic game $G$.

5. If at least one player in $S$ rejects $z^{S}$, then with probability $\rho$ the state does not change (it remains $\omega=\left(S, b^{N \backslash S}\right)$; we call this "repeat"), and with probability $1-\rho$ the rejected proposer $k$ becomes inactive.

6 . If the rejected proposer becomes inactive, then the randomization $x^{k}$ is performed; let $b^{k} \in A^{k}$ be its realization. The action of player $k$ is fixed from now on at $b^{k} \in A^{k}$, and the new state is $\omega^{\prime}=\left(S \backslash\{k\},\left(b^{N \backslash S}, b^{k}\right)\right)$ : the set of active players is $S \backslash\{k\}$ and the actions of the inactive players are $\left(b^{N \backslash S}, b^{k}\right)$.

7. A new round is started (i.e., one goes back to step 1), with the state being the same $\omega$ in case of repeat, and $\omega^{\prime}$ from step 6 otherwise.

\subsection{Outcomes and Equilibria}

We are interested in the (subgame-)perfect equilibria of the PC procedure that are, in addition, as simple as possible, namely, stationary. This means that the decisions of the players depend only on the payoff-relevant variables, not on the history, nor on the "calendar" time. Formally, for each state $\omega=\left(S, b^{N \backslash S}\right)$ and proposer $k \in S$, the announcement $\left(z^{S}, x^{k}\right)$ of player $k$ depends only on $S, b^{N \backslash S}$, and $k$; and the decision of each player $i \in S \backslash k$ to accept or reject depends only on $S, b^{N \backslash S}, k, z^{S}, x^{k}$, and $i$. Stationary subgame-perfect equilibria will be called SP equilibria for short.

For simplicity, we will assume that each player uses the tie-breaking rule of accepting a proposal when indifferent (i.e., when accepting and rejecting gives the same expected payoff).

The play of the PC procedure ends with probability one (because $\rho<1$ ); its end result is an $N$-tuple of actions $a \in A$ in the original game $G$ (see step 4 in the PC procedure), which we call the final $N$-tuple of actions. This final $a$ is random: It depends on the randomizations of nature (e.g., selecting the proposers and repeating or not after rejection) and of the players themselves.

Fix an $N$-tuple of stationary strategies $\sigma=\left(\sigma^{i}\right)_{i \in N}$.

For each state $\omega=\left(S, b^{N \backslash S}\right)$, let $\alpha_{\omega} \in \Delta(A)$ denote the probability distribution of the final $N$-tuple of actions in the subgame starting from state $\omega$. Because the actions of the players outside $S$ are fixed at $b^{N \backslash S}$, the randomness affects only the actions of the players in $S$, and so $\alpha_{\omega}=\zeta_{\omega}^{S} \times\left\{b^{N \backslash S}\right\}$ for some $\zeta_{\omega}^{S} \in \Delta\left(A^{S}\right)$. We refer to $\zeta_{\omega}^{S}$ as the outcome of state $\omega$. Similarly, $\zeta_{\omega, k}^{S} \in \Delta\left(A^{S}\right)$ denotes the 
probability distribution of the final actions of $S$ after $k \in S$ has been selected as proposer; we call it the outcome of state $\omega=\left(S, b^{N \backslash S}\right)$ and proposer $k$. Because the proposer is equally likely to be any member of $S$, we have ${ }^{1}$

$$
\zeta_{\omega}^{S}=\frac{1}{|S|} \sum_{k \in S} \zeta_{\omega, k}^{S}
$$

for every state $\omega=\left(S, b^{N \backslash S}\right)$. The collection of outcomes $\zeta_{\omega}^{S}$ and $\zeta_{\omega, k}^{S}$ for all possible states and proposers (i.e., $\omega=\left(S, b^{N \backslash S}\right)$ for $S \subset N, b^{N \backslash S} \in A^{N \backslash S}$, and $k \in S$ ) will be called an outcome configuration (obtained from $\sigma$ ) and will be denoted $\zeta$.

For every $k \in S$ and every $b^{k} \in A^{k}$, let $\left(\omega \| b^{k}\right):=\left(S \backslash k,\left(b^{N \backslash S}, b^{k}\right)\right)$ denote the state obtained from $\omega$ when $k$ becomes inactive and his action is fixed at $b^{k}$; and, for every $x^{k} \in \Delta\left(A^{k}\right)$ let

$$
\eta_{\omega, k}^{S}\left(x^{k}\right):=\sum_{b^{k} \in A^{k}} x^{k}\left(b^{k}\right)\left(\zeta_{\left(\omega \| b^{k}\right)}^{S \backslash k} \times\left\{b^{k}\right\}\right) \in \Delta\left(A^{S}\right)
$$

be the expected outcome for $S$ following the implementation of the threat $x^{k}$. We will say that an announcement $\left(z^{S}, x^{k}\right) \in \Delta\left(A^{S}\right) \times \Delta\left(A^{k}\right)$ of player $k$ is "acceptable" if, when the continuation is according to $\zeta$, each responder's payoff from accepting $z^{S}$ is no less than his payoff from rejecting it, that is,

$$
u^{i}\left(z^{S}, b^{N \backslash S}\right) \geq \rho u^{i}\left(\zeta_{\omega}^{S}, b^{N \backslash S}\right)+(1-\rho) u^{i}\left(\eta_{\omega, k}^{S}\left(x^{k}\right), b^{N \backslash S}\right)
$$

for every $i \in S \backslash k$ (recall that after rejection the state remains $\omega$ with probability $\rho$, and with probability $1-\rho$ player $k$ becomes inactive and his threat is realized). Let $Y \equiv Y_{\omega, k}(\zeta)$ denote the set of acceptable announcements of $k$ :

$$
Y:=\left\{\left(z^{S}, x^{k}\right) \in \Delta\left(A^{S}\right) \times \Delta\left(A^{k}\right):(3) \text { holds for every } i \in S \backslash k\right\},
$$

and let $Y^{*} \equiv Y_{\omega, k}^{*}(\zeta)$ be the set of those acceptable announcements that maximize the payoff of the proposer ${ }^{2} k$ :

$$
Y^{*}:=\arg \max _{\left(z^{S}, x^{k}\right) \in Y} u^{k}\left(z^{S}, b^{N \backslash S}\right) .
$$

Finally, denote by $Z \equiv Z_{\omega, k}(\zeta)$ and $Z^{*} \equiv Z_{\omega, k}^{*}(\zeta)$ the projections of the sets $Y$ and $Y^{*}$, respectively, on the $z^{S}$-coordinate:

$$
\begin{aligned}
Z & :=\left\{z^{S} \in \Delta\left(A^{S}\right):\left(z^{S}, x^{k}\right) \in Y \text { for some } x^{k} \in \Delta\left(A^{k}\right)\right\} ; \quad \text { and } \\
Z^{*} & :=\left\{z^{S} \in \Delta\left(A^{S}\right):\left(z^{S}, x^{k}\right) \in Y^{*} \text { for some } x^{k} \in \Delta\left(A^{k}\right)\right\} .
\end{aligned}
$$

1. For a finite set $Z$, we denote by $|Z|$ the number of elements of $Z$.

2. We write arg max for the set of maximizers. 
We claim that the SP equilibrium conditions on the outcome configuration $\zeta$ can be stated as

$$
\zeta_{\omega, k}^{S} \in Z_{\omega, k}^{*}(\zeta)
$$

for every state $\omega=\left(S, b^{N \backslash S}\right)$ and $k \in S$. Note that relationship (4) is a fixedpoint-type condition.

PROPOSITION 1. An outcome configuration $\zeta$ is obtained from an SP equilibrium of the PC procedure if and only if $\zeta$ satisfies condition (4) for all states $\omega=$ $\left(S, b^{N \backslash S}\right)$ and $k \in S$.

Proof. Let $\zeta$ satisfy the condition (4). Define an $N$-tuple of strategies $\sigma$ as follows: In state $\omega=\left(S, b^{N \backslash S}\right)$, when $k \in S$ is the proposer he announces an element $\left(\tilde{z}^{S}, \tilde{x}^{k}\right) \in Y_{\omega, k}^{*}$ with $\tilde{z}^{S}=\zeta_{\omega, k}^{S}$, and when $i \in S$ is a responder he accepts a proposal $z^{S}$ if and only if condition (3) holds. It is straightforward to verify (using a "one-deviation property" as in, e.g., Osborne and Rubinstein (1994) Lemma 98.2) that $\sigma$ constitutes an SP equilibrium, and its outcome configuration is precisely the given $\zeta$.

Conversely, let $\sigma$ be an SP equilibrium with outcome configuration $\zeta$. Take a state $\omega=\left(S, b^{N \backslash S}\right)$ and a proposer $k \in S$, and consider a single deviation from $\sigma$, at this point only, by player $k$. We claim that the set of outcomes that $k$ can induce is precisely $Z \equiv Z_{\omega, k}(\zeta)$. Indeed, an announcement $\left(z^{S}, x^{k}\right)$ yields acceptance by all players in $S \backslash k$ if and only if condition (3) holds, namely, if and only if $\left(z^{S}, x^{k}\right) \in Y$ (by the equilibrium requirement when there is strict inequality, and by the tie-breaking rule when there is equality). When $\left(z^{S}, x^{k}\right)$ is rejected the continuation outcome is $\bar{z}^{S}:=\rho \zeta_{\omega}^{S}+(1-\rho) \eta_{\omega, k}^{S}\left(x^{k}\right)$, and here too we have $\left(\bar{z}^{S}, x^{k}\right) \in Y$ (condition (3) holds as equalities). Therefore we have shown that $Z$, the projection of $Y$ on the $z^{S}$-coordinate, is indeed the set of all possible outcomes that $k$ can induce at this point (whether there is acceptance or rejection). But $k$ maximizes his payoff (since $\sigma$ is an equilibrium), from which condition (4) follows.

We note two simple but useful facts. For every state $\omega=\left(S, b^{N \backslash S}\right) \operatorname{let}^{3}$

$$
C(\omega):=\left\{u^{S}\left(z^{S}, b^{N \backslash S}\right) \in \mathbb{R}^{S}: z^{S} \in \Delta\left(A^{S}\right)\right\}
$$

be the set of feasible payoff vectors for the coalition $S$ at $\omega$ (i.e., given the fixed actions $b^{N \backslash S} \in A^{N \backslash S}$ of the players outside $S$ ).

3. We write $u^{S}(z)$ for the payoff vector $\left(u^{i}(z)\right)_{i \in S} \in \mathbb{R}^{S}$. 
Lemma 1. Let $\sigma$ be an SP equilibrium with outcome configuration $\zeta$. For every state $\omega=\left(S, b^{N \backslash S}\right)$ and $k \in S$ :

(i) $Y_{\omega, k}(\zeta)$ is a nonempty polytope; and

(ii) there does not exist $c \in C(\omega)$ such that $c \geq u^{S}\left(\zeta_{\omega, k}^{S}\right)$ with strict inequality $c^{k}>u^{k}\left(\zeta_{\omega, k}^{S}\right)$ in the $k$-th coordinate. ${ }^{4}$

Proof. (i) The set $Y$ is nonempty because for every $x^{k} \in \Delta\left(A^{k}\right)$ we have $\left(\bar{z}^{S}, x^{k}\right) \in Y$, where $\bar{z}^{S}:=\rho \zeta_{\omega}^{S}+(1-\rho) \eta_{\omega, k}^{S}\left(x^{k}\right)$ (condition (3) holds as equalities). It is a convex polytope because it is defined by the finitely many inequalities (3) that are linear in $z^{S}$ and $x^{k}$ (the outcomes $\zeta^{S}$ and $\zeta^{S \backslash k}$ are fixed).

(ii) Assume that there is $z^{S} \in \Delta\left(A^{S}\right)$ such that $c=u^{S}\left(z^{S}, b^{N \backslash S}\right) \in C(\omega)$ satisfies $c \geq u^{S}\left(\zeta_{\omega, k}^{S}\right)$ and $c^{k}>u^{k}\left(\zeta_{\omega, k}^{S}\right)$. Replacing $\zeta_{\omega, k}^{S}$ by $z^{S}$ preserves the inequalities (3): Indeed, the left-hand side increases by $\delta:=c^{i}-u^{i}\left(\zeta_{\omega, k}^{S}\right) \geq 0$, whereas the right-hand side increases by less than $\delta$, specifically $(\rho /|S|) \delta$; see equation (1). Therefore $\left(z^{S}, x^{k}\right) \in Y$ is also an acceptable announcement (with the threat $x^{k}$ unchanged), but the payoff of $k$ is strictly higher there, which contradicts condition (4).

\section{General Results}

In this section we prove two general results of a standard type. First, we show that SP equilibria exist; and second, that as the probability of repeat gets close to 1 -that is, as the "cost of delay" goes to zero-the SP equilibrium outcomes approach Pareto efficiency.

\subsection{Existence}

Proposition 2. There exists an SP equilibrium.

Proof. We proceed by induction on $S$. For $|S|=1$, say $S=\{i\}$, the strategy of player $i$ in state $\left(\{i\}, b^{N \backslash i}\right)$ consists of choosing $z^{i} \in$ $\arg \max _{x^{i} \in \Delta\left(A^{i}\right)} u^{i}\left(x^{i}, b^{N \backslash i}\right)$.

Let the state be $\omega=\left(S, b^{N \backslash S}\right)$, and assume that equilibrium strategies and outcomes have been determined for all states $\omega^{\prime}=\left(S^{\prime}, b^{N \backslash S^{\prime}}\right)$ with $S^{\prime} \subsetneq S$. For each $c \in C(\omega)$ (the set of feasible payoff vectors for $S$ ) and $k \in K$, let

$$
\begin{aligned}
\Phi_{k}(c):= & \left\{\left(z^{S}, x^{k}\right) \in \Delta\left(A^{S}\right) \times \Delta\left(A^{k}\right):\right. \\
& \left.u^{i}\left(z^{S}, b^{N \backslash S}\right)-(1-\rho) u^{i}\left(\eta_{\omega, k}^{S}\left(x^{k}\right)\right) \geq \rho c^{i} \text { for all } i \in S \backslash k\right\},
\end{aligned}
$$

4. This implies that $u^{S}\left(\zeta_{\omega, k}^{S}\right)$ is weakly Pareto efficient in $C(\omega)$. 
where $\eta_{\omega, k}^{S}\left(x^{k}\right)$ is defined in equation (2) (based on the $\zeta_{\left(\omega \| b^{k}\right)}^{S \backslash k}$, which have already been determined by induction). The set $\Phi_{k}(c)$ is nonempty (take $z^{S} \in \Delta\left(A^{S}\right)$ with $u^{S}\left(z^{S}, b^{N \backslash S}\right)=c$; then $\left(\tilde{z}^{S}, x^{k}\right) \in \Phi_{k}(c)$ where $\left.\tilde{z}^{S}=\rho z^{S}+(1-\rho) \eta_{\omega, k}^{S}\left(x^{k}\right)\right)$ and is a convex polytope (note that $\eta_{\omega, k}^{S}\left(x^{k}\right)$ is linear in $x^{k}$ ); the correspondence $\Phi_{k}$ is continuous on $C(\omega)$ (by Lemma 2 below). Therefore

$$
\Phi_{k}^{*}(c):=\arg \max _{\left(z^{S}, x^{k}\right) \in \Phi_{k}(c)} u^{k}\left(z^{S}, b^{N \backslash S}\right)
$$

is a nonempty, convex-valued, and upper-semicontinuous correspondence (the latter by the Maximum Theorem because $u^{k}$ is linear and thus continuous, and $\Phi_{k}$ is a continuous correspondence; see, e.g., Hildenbrand (1974), Corollary to Theorem B.III.4). Hence the same holds for the correspondences $\Psi_{k}$ and $\Psi$, defined by

$$
\begin{aligned}
\Psi_{k}(c) & :=\left\{u^{S}\left(z^{S}, b^{N \backslash S}\right):\left(z^{S}, x^{k}\right) \in \Phi_{k}^{*}(c)\right\} \quad \text { and } \\
\Psi(c) & :=\frac{1}{|S|} \sum_{k \in S} \Psi_{k}(c) .
\end{aligned}
$$

We can therefore apply Kakutani's Fixed-Point Theorem (see, e.g., Hildenbrand (1974) C.III (14)) to the correspondence $\Psi$ (with domain $C(\omega)$ ), to obtain $\bar{c} \in C(\omega)$ with $\bar{c} \in \Psi(\bar{c})$. This in turn yields $\bar{c}_{k} \in \Psi_{k}(\bar{c})$ with $\bar{c}=(1 /|S|) \sum_{k \in S} \bar{c}^{k}$, and $\left(\bar{z}^{S}, \bar{x}^{k}\right) \in \Phi_{k}^{*}(\bar{c})$. It is immediate to verify that the announcements $\left(\bar{z}^{S}, \bar{x}^{k}\right)$ for all $k \in S$ constitute equilibrium announcements in state $\omega$. This completes the induction step, and thus proves our claim.

REMARK 1. When $\rho=0$ there is no need to use a fixed-point theorem to prove existence: The SP equilibria can be computed recursively, starting with singleton $S$.

In the proof we have used the following:

Lemma 2. Let $D$ be an $m \times n$ matrix and put $F(w):=\left\{x \in \mathbb{R}^{n}: D x \geq w\right\}$ for every $w \in \mathbb{R}^{m}$. Then $F$ is a continuous correspondence on $W:=\left\{w \in \mathbb{R}^{m}\right.$ : $F(w) \neq \emptyset\}$.

Proof. Upper semicontinuity is immediate. For lower semicontinuity, let $x_{0} \in \mathbb{R}^{n}$ satisfy $D x_{0} \geq w_{0}$, and let $w_{r} \rightarrow w_{0}$ with $w_{r} \in W$ for all $r$; we have to show that for every $r$ there is $x_{r}$ with $D x_{r} \geq w_{r}$. It suffices to consider the case where only one coordinate of $w_{0}$ changes, say, $w_{r}=w_{0}+\left(\delta_{r}, 0, \ldots, 0\right)$. If $\delta_{r} \rightarrow 0^{-}$, then take $x_{r}=x_{0}$. If $\delta_{r} \rightarrow 0^{+}$, then let $x_{1}$ satisfy $D x_{1} \geq w_{1}$ (recall that $w_{1} \in W$ ), and then $x_{r}:=\left(1-\delta_{r} / \delta_{1}\right) x_{0}+\left(\delta_{r} / \delta_{1}\right) x_{1}$ satisfies $D x_{r} \geq w_{0}+\left(\delta_{r}, 0, \ldots, 0\right)=w_{r} . \square$ 


\subsection{Pareto Efficiency}

In equilibrium, every individual proposal $\zeta_{\omega, k}^{S}$ is (weakly) Pareto efficient (see Lemma 1 (ii)). Therefore the outcomes $\zeta_{\omega}^{S}$ may fail to be efficient only if the Pareto-efficient boundary is not a hyperplane and the individual proposals of different proposers are different (see equation (1)). However, if $\rho$ is close to $1-$ that is, the "cost of delay" is small—-then the early-proposer's advantage will be small, and so the individual proposals will be similar and their average almost Pareto efficient.

To see this, let $\zeta(\rho)$ be an SP equilibrium outcome for the PC bargaining procedure with parameter $\rho$-we will refer to it as the PC " $\rho$-procedure." Consider a limit point $\bar{\zeta}$ of $\zeta(\rho)$ as $\rho \rightarrow 1$ (i.e., there is a sequence $\rho_{m} \rightarrow 1$ such that $\zeta\left(\rho_{m}\right) \rightarrow \bar{\zeta}$ as $\left.m \rightarrow \infty\right)$. Then we have the following theorem.

THeOREM 1. Let $\bar{\zeta}=\left(\bar{\zeta}_{\omega}^{S}\right)_{\omega}$ be a limit point as $\rho \rightarrow 1$ of SP equilibrium outcomes $\zeta(\rho)=\left(\zeta_{\omega}^{S}(\rho)\right)_{\omega}$ of the PC $\rho$-procedures. Then for every state $\omega=$ $\left(S, b^{N \backslash S}\right)$ the limit outcome $\bar{\zeta}_{\omega}^{S}$ in state $\omega$ is Pareto efficient for $S$ given $b^{N \backslash S}$.

Proof. Assume for simplicity that $\zeta(\rho) \rightarrow \bar{\zeta}$ as $\rho \rightarrow 1$ (otherwise restrict the arguments to the sequence $\rho_{m}$ with $\left.\zeta\left(\rho_{m}\right) \rightarrow \bar{\zeta}\right)$. Put $g_{\omega, k} \equiv g_{\omega, k}(\rho):=$ $u^{S}\left(\zeta_{\omega, k}^{S}(\rho), b^{N \backslash S}\right), g_{\omega} \equiv g_{\omega}(\rho):=u^{S}\left(\zeta_{\omega}^{S}(\rho), b^{N \backslash S}\right)$, and $\bar{g}_{\omega}:=u^{S}\left(\bar{\zeta}_{\omega}^{S}, b^{N \backslash S}\right)$ for all $k \in S$; thus $g_{\omega} \rightarrow \bar{g}_{\omega}$ as $\rho \rightarrow 1$. Let $M$ be a bound on all possible payoffs of all players; for each $\rho$ we have

$$
g_{\omega, k}^{i} \geq \rho g_{\omega}^{i}+(1-\rho) u^{i}\left(\eta_{\omega, k}^{S}\left(x^{k}\right), b^{N \backslash S}\right) \geq g_{\omega}^{i}-(1-\rho) 2 M
$$

(for $i \neq k$ it follows from condition (4), the definition of $Y$, and condition (3); for $i=k$, from condition (4) together with $\left(\tilde{z}^{S}, x^{k}\right) \in Y$ for $\tilde{z}^{S}:=\rho \zeta_{\omega}^{S}+(1-$ $\left.\rho) \eta_{\omega, k}^{S}\left(x^{k}\right)\right)$. Now $g_{\omega}^{i}=(1 /|S|) \sum_{k \in S} g_{\omega, k}^{i}$ by equation (1), and so adding the inequalities (5) for all $k$ except some $k_{0} \in S$ (keep $i$ fixed) yields

$$
|S| g_{\omega}^{i}-g_{\omega, k_{0}}^{i} \geq(|S|-1)\left(g_{\omega}^{i}-(1-\rho) 2 M\right),
$$

or

$$
g_{\omega, k_{0}}^{i} \leq g_{\omega}^{i}+(1-\rho)(|S|-1) 2 M \leq g_{\omega}^{i}+(1-\rho) 2 M|N|
$$

for all $k_{0} \in S$. Thus

$$
-(1-\rho) 2 M \leq g_{\omega, k}^{i}-g_{\omega}^{i} \leq(1-\rho) 2 M|N|
$$

(replace $k_{0}$ by $k$ to get the second inequality, and recall condition (5) for the first); hence, as $\rho \rightarrow 1$, we get $g_{\omega, k}^{i}-g_{\omega}^{i} \rightarrow 0$, which, because $g_{\omega}^{i} \rightarrow \bar{g}_{\omega}^{i}$, implies that $g_{\omega, k}^{i} \rightarrow \bar{g}_{\omega}^{i}$ for all $i, k \in S$. 
If $\bar{g}_{\omega}$ is not Pareto efficient in $C(\omega)$, then there exist $k \in S$ and $c \in C(\omega)$ such that $c \geq \bar{g}_{\omega}$, with strict inequality in the $k$-th coordinate. Then $c(\rho):=$ $g_{\omega, k}(\rho)+(1 / 2)\left(c-\bar{g}_{\omega}\right)$ satisfies $c(\rho) \geq g_{\omega, k}(\rho)$, with strict inequality in the $k$-th coordinate; also, for $\rho$ close enough to 1 , we have $c(\rho) \in C$ (use the fact that $C$ is determined by finitely many linear inequalities and $\left.g_{\omega, k}(\rho) \rightarrow \bar{g}_{\omega}\right)$. But this contradicts Lemma 1 (ii).

\section{Two Reference Cases: Two Players and Transferable Utility}

In this section we spell out the nature of our solution for two simple, classical cases.

\subsection{Two-Person Games}

The SP equilibria of the PC procedure relate very directly to the Nash bargaining solution for the case of two players (cf. Hart and Mas-Colell (1996a); see also Houba and Bolt (2002) for more on two-person bargaining games).

Given a two-person game $G$ with $N=\{1,2\}$, for each player $i \in N$ let $q^{i}$ be the payoff level that the other player $j \neq i$ can hold $i$ to, by using pure strategies; namely,

$$
q^{i}:=\min _{a^{j} \in A^{j}} \max _{a^{i} \in A^{i}} u^{i}\left(a^{i}, a^{j}\right)
$$

Let

$$
D:=\left\{u^{N}(z): z \in \Delta(A)\right\}=\operatorname{co}\left\{\left(u^{1}(a), u^{2}(a)\right): a \in A\right\}
$$

be the set of feasible payoff vectors. $(D, q)$ is called a two-person pure bargaining problem, with $D$ the set of "feasible agreements" and $q$ the "disagreement point," if $q \in D$ and there exists $d \in D$ such that $d^{1}>q^{1}$ and $d^{2}>q^{2}$ (see Nash 1950).

Proposition 3. Let $G$ be a two-person strategic game such that $(D, q)$ is a pure bargaining problem. If $\bar{\zeta}^{N}$ is a limit point as $\rho \rightarrow 1$ of SP equilibrium outcomes, then $u^{N}\left(\bar{\zeta}^{N}\right)$ is the Nash bargaining solution of $(D, q)$.

Proof. $\bar{\zeta}^{N}$ is Pareto efficient by Theorem 1. If the Pareto boundary $\partial_{+} D$ of $D$ contains only $u^{N}\left(\bar{\zeta}^{N}\right)$ then we are done, because the Nash solution is Pareto efficient. If not, assume first that $u^{N}\left(\bar{\zeta}^{N}\right)$ is an interior point of $\partial_{+} D$. Let $\omega=(N, \cdot)$ be the starting state; because $u^{N}\left(\zeta_{\omega, 1}^{N}(\rho)\right)$ is weakly Pareto efficient and it converges to $u^{N}\left(\bar{\zeta}^{N}\right)$, it follows that $u^{N}\left(\zeta_{\omega, 1}^{N}(\rho)\right) \in \partial_{+} D$ for all $\rho$ close enough to 1 . For every $b^{1} \in A^{1}$, the payoff that player 2 gets in the state $\left(\{2\}, b^{1}\right)$ is 
$v^{2}\left(b^{1}\right):=\max _{a^{2} \in A^{2}} u^{2}\left(a^{2}, b^{1}\right)$, and so condition (4) says that $u^{1}\left(\zeta_{\omega, 1}^{N}(\rho)\right) \max$ imizes $u^{1}\left(z^{N}\right)$ subject to $u^{2}\left(z^{N}\right) \geq \rho u^{2}\left(\zeta^{N}(\rho)\right)+(1-\rho) v^{2}\left(b^{1}\right)$. Therefore any $b^{1} \in A^{1}$ that has positive probability in the threat $x^{1} \in \Delta\left(A^{1}\right)$ that is used by player 1 must make $v^{2}\left(b^{1}\right)$ as small as possible (here we use the Pareto efficiency of $\left.u^{N}\left(\zeta_{\omega, 1}^{N}(\rho)\right) \in \partial_{+} D\right)$; but $\min _{b^{1} \in A^{1}} v^{2}\left(b^{1}\right)=q^{2}$, and so $u^{1}\left(\zeta_{\omega, 1}^{N}(\rho)\right)$ maximizes $u^{1}\left(z^{N}\right)$ subject to $u^{2}\left(z^{N}\right)=\rho u^{2}\left(\zeta^{N}(\rho)\right)+(1-\rho) q^{2}$. A similar argument applies when we interchange the two players; from this it follows by standard arguments that the limit $u^{N}\left(\bar{\zeta}^{N}\right)$ of $u^{N}\left(\zeta^{N}(\rho)\right)$ as $\rho \rightarrow 1$ is precisely the Nash bargaining solution of $(D, q)$; see for example Hart and Mas-Colell (1996a, Theorem 3). Finally, if $u^{N}\left(\bar{\zeta}^{N}\right)$ is an extreme point of $\partial_{+} D$-it minimizes, say, player 1 's payoff and maximizes player 2's payoff on $\partial_{+} D$-then the above argument applies only to $\zeta_{\omega, 1}^{N}(\rho)$; for $\zeta_{\omega, 2}^{N}(\rho)$ we get some $\hat{q}^{1}(\rho) \geq q^{1}$ such that $u^{2}\left(\zeta_{\omega, 2}^{N}(\rho)\right)$ maximizes $u^{2}\left(z^{N}\right)$ subject to $u^{1}\left(z^{N}\right)=\rho u^{1}\left(\zeta^{N}(\rho)\right)+(1-\rho) \hat{q}^{1}(\rho)$. Therefore $u^{N}\left(\bar{\zeta}^{N}\right)$ is the Nash bargaining solution of $\left(D,\left(\hat{q}^{1}, q^{2}\right)\right)$ where $\hat{q}^{1} \geq q^{1}$ is a limit point of $\hat{q}^{1}(\rho)$ as $\rho \rightarrow 1$; given that $u^{N}\left(\bar{\zeta}^{N}\right)$ is that extreme point of $\partial_{+} D$ where player 1's payoff is minimal, it easily follows that $u^{N}\left(\bar{\zeta}^{N}\right)$ is also the solution of $(D, q)$.

Remark 2. One could well have $q \notin D$; for example, in the "matching pennies" game, $D$ is the line segment connecting $(1,-1)$ and $(-1,1)$, and $q=(1,1)$. In this case we have a "reverse pure bargaining problem" and $u^{N}\left(\bar{\zeta}^{N}\right)$ is its solution (see the discussion in Section 4 of Hart and Mas-Colell $(1996 \mathrm{a}) ; u^{N}\left(\bar{\zeta}^{N}\right)$ in this example is $(0,0))$.

\subsection{Transferable Utility}

Given the game $G$, the individually rational level in pure actions of player $i$ is

$$
r^{i}:=\max _{a^{i} \in A^{i}} \min _{a^{N \backslash i} \in A^{N \backslash i}} u^{i}\left(a^{i}, a^{N \backslash i}\right)=\max _{a^{i} \in A^{i} z^{N \backslash i} \in \Delta\left(A^{N \backslash i}\right)} u^{i}\left(a^{i}, z^{N \backslash i}\right) ;
$$

this is the maximum that $i$ can guarantee by using pure strategies. The payoff of player $i$ in any equilibrium of the PC procedure will always be at least $r^{i}$ (the following strategy $\sigma^{i}$ guarantees $r^{i}$ : When $i$ is the proposer his threat is some $a^{i} \in A^{i}$ where the max is attained-namely, $\min _{a^{N \backslash i} \in A^{N \backslash i}} u^{i}\left(a^{i}, a^{N \backslash i}\right)=r^{i}$ holds - and when he is the responder he never accepts any payoff less than $\left.{ }^{5} r^{i}\right)$. Thus, only payoff vectors $c=\left(c^{i}\right)_{i \in N}$ that are individually rational-i.e., $c^{i} \geq r^{i}$ for each $i-$ matter.

5. The intuitive reason why $r^{i}$ is based on pure actions of $i$ (rather than mixed ones) is that if $i$ 's proposal is rejected then the randomization in his threat $x^{i} \in \Delta\left(A^{i}\right)$ is realized, and from then on $i$ is fixed at a pure action that is known to everyone; see Section 8 (a)(i). 
We say that the game $G$ is a strategic game with transferable utility (a "strategic TU game" for short) if, for every state $\omega=\left(S, b^{N \backslash S}\right)$, that is, for every $S \subset N$ and $b^{N \backslash S} \in A^{N \backslash S}$, there exists a number $v(\omega) \equiv v\left(S, b^{N \backslash S}\right)$ such that every Pareto efficient and individually rational payoff vector $c$ in $C(\omega):=\left\{u^{S}\left(z^{S}, b^{N \backslash S}\right): z^{S} \in \Delta\left(A^{S}\right)\right\}$ satisfies

$$
\sum_{i \in S} c^{i}=v(\omega) \equiv v\left(S, b^{N \backslash S}\right) .
$$

If $G$ is a strategic TU game, then the SP equilibria of the PC procedure become relatively simple to determine. In particular, no fixed point is needed and the computation is not recursive, as the threats can be determined independently for each coalition $S$.

For every state $\omega=\left(S, b^{N \backslash S}\right)$, proposer $k \in S$, and mixed action $x^{k} \in$ $\Delta\left(A^{k}\right)$, extend the definition of $v(\cdot)$ to mixed actions:

$$
v\left(S \backslash k,\left(b^{N \backslash S}, x^{k}\right)\right):=\sum_{b^{k} \in A^{k}} x^{k}\left(b^{k}\right) v\left(S \backslash k,\left(b^{N \backslash S}, b^{k}\right)\right),
$$

and define

$$
\begin{aligned}
\tau_{\omega, k} & :=\min _{x^{k} \in \Delta\left(A^{k}\right)} v\left(S \backslash k,\left(b^{N \backslash S}, x^{k}\right)\right)=\min _{b^{k} \in A^{k}} v\left(S \backslash k,\left(b^{N \backslash S}, b^{k}\right)\right) ; \quad \text { and } \\
X_{\omega}^{k} & :=\arg \min _{x^{k} \in \Delta\left(A^{k}\right)} v\left(S \backslash k,\left(b^{N \backslash S}, x^{k}\right)\right)
\end{aligned}
$$

(note that $X_{\omega}^{k}$ consists of all pure actions $b^{k} \in A^{k}$ that are minimizers of $v\left(S \backslash k,\left(b^{N \backslash S}, b^{k}\right)\right)$, along with all their probabilistic mixtures). Finally, put

$$
D^{k} v(\omega):=v(\omega)-\tau_{\omega, k}=v\left(S, b^{N \backslash S}\right)-\min _{b^{k} \in A^{k}} v\left(S \backslash k,\left(b^{N \backslash S}, b^{k}\right)\right) ;
$$

this is the "marginal contribution" of player $k \in S$ in state $\omega=\left(S, b^{N \backslash S}\right)$.

A threat configuration $\boldsymbol{x}=\left(x_{\omega}^{k}\right)_{\omega, k}$ is a collection of mixed actions $x_{\omega}^{k} \in$ $\Delta\left(A^{k}\right)$ for every state $\omega=\left(S, b^{N \backslash S}\right)$ and every $k \in S$; every $N$-tuple of stationary pure strategies $\sigma$ generates such an $x$ : take the second coordinate of the announcements (in state $\omega$ a proposer $k$ announces $\left(\zeta_{\omega, k}^{S}, x_{\omega}^{k}\right) \in \Delta\left(A^{S}\right) \times \Delta\left(A^{k}\right)$ ).

Next, let $\Pi$ be the set of all $|N|$ ! orders of the players. For each order $\pi=$ $\left(i_{1}, i_{2}, \ldots, i_{N}\right) \in \Pi$ and threat configuration $\boldsymbol{x}$ define a probability distribution $Q_{\pi} \equiv Q_{\pi}^{x}$ on $A$ as follows:

$$
Q_{\pi}(b):=\prod_{m=1}^{N} x_{\omega_{m}}^{i_{m}}\left(b^{i_{m}}\right)
$$


for every $b \in A$, where, for each $m=1,2, \ldots, N$, we put $S_{m}:=$ $\left\{i_{m}, i_{m+1}, \ldots, i_{N}\right\}, b^{N \backslash S_{m}}:=\left(b^{i_{1}}, b^{i_{2}}, \ldots, b^{i_{m-1}}\right)$, and $\omega_{m}:=\left(S_{m}, b^{N \backslash S_{m}}\right)$. Taking the order $\pi \in \Pi$ to be random, with all $|N|$ ! orders equally likely, yields a joint probability distribution $Q \equiv Q^{x}$ on $\Pi \times A$ :

$$
Q(\pi, b):=\frac{1}{N !} Q_{\pi}(b)
$$

for every $\pi \in \Pi$ and $b \in A$. For each $(\pi, b) \in \Pi \times A$ and player $i \in N$, let $P_{\pi}^{i}$ denote the set of predecessors of $i$ in the order $\pi$, and let $\omega_{\pi, b}^{i}=\left(N \backslash P_{\pi}^{i}, b^{P_{\pi}^{i}}\right)$ be the state where each predecessor $j \in P_{\pi}^{i}$ has his action fixed at the corresponding $b^{j}$.

Finally, let

$$
\phi^{i}:=\mathbf{E}\left[D^{i} v\left(\omega_{\pi, b}^{i}\right)\right]
$$

be the "expected marginal contribution" of player $i$ to his predecessors, where $\mathbf{E}$ denotes expectation with respect to the distribution $Q^{x}$ on $\Pi \times A$, and $\omega_{\pi, b}^{i}$ is the state determined as above.

Proposition 4. Let $G$ be a strategic TU game with associated function $v$. If $\sigma$ is an SP equilibrium of the PC $\rho$-procedure, then the resulting threat configuration $\boldsymbol{x}=\left(x_{\omega}^{k}\right)_{\omega, k}$ satisfies $x_{\omega}^{k} \in X_{\omega, k}$ for every $\omega$ and $k$ (see equations (7) and (8)). Conversely, for each $\boldsymbol{x}=\left(x_{\omega}^{k}\right)_{\omega, k}$ satisfying $x_{\omega}^{k} \in X_{\omega, k}$ for every $\omega$ and $k$, there exists an SP equilibrium $\sigma$ with this threat configuration. Moreover, the payoff of each player $i \in N$ in that equilibrium $\sigma$ equals $\phi^{i}$ of formula (12), where the probability distribution $Q \equiv Q^{\boldsymbol{x}}$ is determined by the collection $\boldsymbol{x}=\left(x_{\omega}^{k}\right)_{\omega, k}$ according to equations (10) and (11).

Remark 3. The threats $x_{\omega}^{k}$ and the payoffs do not depend on $\rho$. Moreover, the determination of any set $X_{\omega}^{k}$ can be done independently of any other such set. This holds here, in the TU case, but not in general, where optimal threats are determined recursively (i.e., one needs to determine first the optimal threats and proposals at all states that correspond to the subgames of $\omega$ ).

REMARK 4. In every state, the payoffs and proposals are determined in the same manner, by considering only the appropriate subgame.

Proof of Proposition 4. Let $\sigma$ be an SP equilibrium. For each $\omega=\left(S, b^{N \backslash S}\right)$ and $k \in S$, let $g_{\omega}:=u^{S}\left(\zeta_{\omega}^{S}, b^{N \backslash S}\right)$ and $g_{\omega, k}:=u^{S}\left(\zeta_{\omega, k}^{S}, b^{N \backslash S}\right)$; because $g_{\omega, k}$ is individually rational and Pareto efficient in $C(\omega)$ (recall Lemma 1 (ii)), equation (6) implies that

$$
\sum_{i \in S} g_{\omega, k}^{i}=v(\omega)
$$


Therefore, by equation (1), the same holds for $g_{\omega}$ :

$$
\sum_{i \in S} g_{\omega}^{i}=v(\omega)
$$

Moreover, equation (6) implies that maximizing the $k$-th coordinate $g_{\omega, k}^{k}$ is equivalent to minimizing all the other coordinates $g_{\omega, k}^{i}$, and so condition (3) is satisfied as equalities, that is, for every $i \in S \backslash k$,

$$
g_{\omega, k}^{i}=\rho g_{\omega}^{i}+(1-\rho) u^{i}\left(\eta_{\omega, k}^{S}\left(x_{\omega}^{k}\right)\right) .
$$

Summing this over $i \in S \backslash k$ yields

$$
v(\omega)-g_{\omega, k}^{k}=\rho\left(v(\omega)-g_{\omega}^{k}\right)+(1-\rho) t
$$

where

$$
\begin{aligned}
t & :=\sum_{b^{k} \in A^{k}} x_{\omega}^{k}\left(b^{k}\right) \sum_{i \in S \backslash k} u^{i}\left(\zeta_{\left(\omega \| b^{k}\right)}^{S \backslash k},\left(b^{N \backslash S}, b^{k}\right)\right) \\
& =\sum_{a^{k} \in A^{k}} x_{\omega}^{k}\left(b^{k}\right) v\left(S \backslash k,\left(b^{N \backslash S}, b^{k}\right)\right)
\end{aligned}
$$

(we have used equation (14) for $S \backslash k$ ). Rewrite equation (16) as

$$
(1-\rho)(v(\omega)-t)=g_{\omega, k}^{k}-\rho g_{\omega}^{k}=\left(1-\frac{\rho}{|S|}\right) g_{\omega, k}^{k}-\frac{\rho}{|S|} \sum_{j \in S \backslash k} g_{\omega, j}^{k} .
$$

Therefore, in order to maximize $g_{\omega, k}^{k}$ (i.e., to satisfy condition (4)), one must minimize $t$ (the other terms are fixed here). But $t$ depends only on the threat $x_{\omega}^{k}$ (and the given function $v$ ), and so $t=\tau_{\omega, k}$ and $x_{\omega}^{k} \in X_{\omega}^{k}$; therefore (see equations (9) and (18)):

$$
g_{\omega, k}^{k}=\rho g_{\omega}^{k}+(1-\rho) D^{k} v(\omega)
$$

Let

$$
h_{\omega, i}^{k}:=u^{k}\left(\eta_{\omega, i}^{S}\left(x_{\omega}^{i}\right)\right)=\sum_{b^{i} \in A^{i}} x_{\omega}^{i}\left(b^{i}\right) g_{\left(\omega \| b^{i}\right)}^{k}
$$

be the payoff of $k$ when $i \neq k$ becomes inactive and his threat $x_{\omega}^{i}$ is implemented; then (by equation (15), interchanging $i$ and $k$ ):

$$
g_{\omega, i}^{k}=\rho g_{\omega}^{k}+(1-\rho) h_{\omega, i}^{k}
$$


Adding this over all $i \neq k$ together with equation (19) yields $|S| g_{\omega}^{k}=\rho|S| g_{\omega}^{k}+$ $(1-\rho)\left(D^{k} v(\omega)+\sum_{i \in S \backslash k} h_{\omega, i}^{k}\right)$, or

$$
g_{\omega}^{k}=\frac{1}{|S|}\left(D^{k} v(\omega)+\sum_{i \in S \backslash k} h_{\omega, i}^{k}\right) .
$$

Substituting equation (20) yields recursively formula (12).

\subsection{Example}

At this point it is useful to analyze a simple example.

Let $N=\{1,2,3\}$, and for each $i \in N$ put $A^{i}=\{0,1\} \times N$, with generic element $a^{i}=\left(c^{i}, d^{i}\right)$ where $c^{i} \in\{0,1\}$ and $d^{i} \in N$. The payoffs are as follows: $u^{i}(a)=u_{1}^{i}(c)+u_{2}^{i}(d)$, where: $u_{1}^{3}(c)=1$ when $c^{1}=c^{2}$ and $c^{3}=1$, and $u_{1}^{3}(c)=0$ otherwise; $u_{1}^{i} \equiv 0$ for $i=1,2$; and $u_{2}^{i}(d):=\left|\left\{j: d^{j}=i\right\}\right|-1$. Thus, according to the $u_{1}^{i}$-part of the payoff functions $u^{i}$, player 3 gets a payoff of 1 when he chooses $c^{3}=1$ and the $c^{i}$-s of players 1 and 2 match; all other payoffs are 0 . The effect of the $u_{2}^{i}$-part is to allow transfers and therefore to make the game TU: $d^{j}=i$ means that player $j$ transfers one unit to player $i$ (i.e., the payoff of $i$ increases by 1 , and that of $j$ decreases by 1 ; note that $d^{j}=j$ means that $j$ makes no transfer).

Consider the PC procedure. The optimal threats are determined by equation (7); this implies that there will never be any transfers (because this only increases the worth of the remaining players after the proposer becomes inactive), and so we will specify only the $c^{i}$ coordinate. Player 3 can always guarantee that the remaining players get 0 (by using the threat $c^{3}=0$ ). The only case where his marginal contribution is not $D^{3} v(\omega)=1-0=1$ is when the threats of the players who became inactive before him made $v$ equal to 0 ; this happens only when player 3 is last, and then the optimal threat of the second player to become inactive, say player 2 , is the opposite of that of player 1 (i.e., $c^{2}=1-c^{1}$ ). Therefore the SP equilibrium payoffs are $(1 / 6,1 / 6,2 / 3)$.

It is interesting to compare this to the more familiar approaches. The von Neumann-Morgenstern coalitional function is $v(N)=1, v(1,3)=1 / 2$ (player 1 plays $c^{1}=0$ and $c^{1}=1$ with $(1 / 2,1 / 2)$ probabilities, and player 3 plays $\left.c^{3}=1\right), v(2,3)=1 / 2$, and $v(S)=0$ otherwise. The Shapley value of this $v$ is $(1 / 4,1 / 4,1 / 2)$.

When going to the Harsanyi coalitional function we get $^{6} v(N)=1$, $v(1)=1 / 4, v(2,3)=3 / 4, v(2)=1 / 4, v(1,3)=3 / 4, v(3)=$

6. Take for example $\{1\}$ vs. $\{2,3\}$. The optimal strategies are $(1 / 2,1 / 2)$ for 1 , vs. $(1 / 2,1 / 2)$ for 2 and $c^{3}=1$ for 3 , which give payoffs of 0 to $\{1\}$ and $1 / 2$ to $\{2,3\}$. Therefore $v(1)=0+(1-0-$ $1 / 2) / 2=1 / 4$ and $v(23)=1 / 2+(1-0-1 / 2) / 2=3 / 4$. 
$1 / 2, v(1,2)=1 / 2$. This is an inessential game, and its value is again $(1 / 4,1 / 4,1 / 2)$.

Interestingly, the SP equilibrium payoffs $(1 / 6,1 / 6,2 / 3)$ seem to reflect better the underlying situation. The payoffs $(1 / 4,1 / 4,1 / 2)$ are what one would expect if $\{1,2\}$ acted as one player, and then split the payoff. But it seems natural that the need to coordinate between 1 and 2 comes at some cost to them, and the payoff vector $(1 / 6,1 / 6,2 / 3)$ captures this better.

\section{Equilibria with Fixed Threats}

Threats are of the essence of the theory we are presenting in this paper. It is because of the strategic linkage across coalitions captured by them that, for example, we cannot in any general and meaningful sense factor our analysis through the coalitional forms of standard cooperative game theory: there is no "worth" of a coalition that is independent of the actions - the threats — of the players outside the coalition.

This difficulty at the foundations of cooperative game theory has, of course, been recognized for a long time. It has led, on the one hand, to the development of extensions of the notion of coalitional form (perhaps the best known are the "games in partition form" of Thrall and Lucas (1963); see Myerson (1977), Maskin (2003), de Clippel and Serrano (2008), and Macho-Stadler, PerezCastrillo, and Wettstein (2007), for more recent work) and on the other to the consideration of particular situations where the classical form could be justified (for example the c-games of Shapley-Shubik; see Shubik (1983), p. 130).

Nonetheless, the discussion of the previous section, and especially expression (12) for the computation of the SP equilibria, suggests a close connection to the cooperative game solutions related to the Shapley (1953) value. In this section we shall throw some light on this connection.

It is certainly the case that along an equilibrium path only the particular actions that may arise as threats matter. But even then the threat of a proposer may depend on the current set of active players and on the threats of the preceding proposers. Still, if the threats happen to be independent of the previous history, we could indeed associate a coalitional form to the particular equilibrium, and we could then analyze how the equilibrium payoffs relate to the cooperative game theory solutions of the coalitional form. This we shall now do.

DEFINITION 1. Let $G$ be a strategic game and $\sigma$ an SP equilibrium of the PC procedure. For every player $k \in N$ let $f^{k} \in A^{k}$ be a pure action of $k$. We say that $\sigma$ has fixed threats $\left(f^{k}\right)_{k \in N}$ if for every $k \in N$, the threat of player $k$ (whenever he is the proposer) is $f^{k}$ in all states where the action of each inactive player $j$ is $f^{j}$ (i.e., in all states of the form $\omega=\left(S, f^{N \backslash S}\right)$ ).

Observe that we in fact require less than the discussion above suggests; namely, the threats need to be fixed only along the "backward induction 
equilibrium paths," and there are no requirements for states $\left(S, b^{N \backslash S}\right)$ with $b^{N \backslash S} \neq f^{N \backslash S}$ (these are the states in which a previous threat that was implemented was different from the corresponding $f^{j}$ ). Also, note that each $f^{k}$ is a pure action, and that having fixed threats is a property of SP equilibria.

Next, given a strategic game $G$ and an SP equilibrium $\sigma$ with fixed threats $\left(f^{k}\right)_{k \in N}$, we say that the NTU coalitional game $\left(N, V_{G, \sigma}\right)$ is derived from $G$ and $\sigma$ if

$$
V_{G, \sigma}(S)=\left\{c \in \mathbb{R}^{S}: c \leq u^{S}\left(z^{S}, f^{N \backslash S}\right) \text { for some } z^{S} \in \Delta\left(A^{S}\right)\right\}
$$

for every coalition $S \subset N$. We have the following result.

Proposition 5. Let $\left(N, V_{G, \sigma}\right)$ be a game that is derived from the strategic game $G$ and the fixed-threat equilibrium $\sigma$. Suppose that $\left(N, V_{G, \sigma}\right)$ is a TU game in the individually rational region. ${ }^{7}$ Then the payoffs induced by $\sigma$ equal the Shapley values of $\left(N, V_{G, \sigma}\right)$ and its subgames. Moreover, if $\rho$ is close to 1 , then the payoffs of the proposals made by the different players will also be close to the Shapley values of $\left(N, V_{G, \sigma}\right)$.

Thus, when $\left(N, V_{G, \sigma}\right)$ is a TU coalitional game-let $v \equiv v_{G, \sigma}$ denote its TU coalitional function-the outcome configuration $\zeta$ of $\sigma$ satisfies

$$
u^{i}\left(\zeta_{\left(S, f^{N \backslash S}\right)}^{S}\right)=\operatorname{Sh}^{i}\left(S, v_{G, \sigma}\right)
$$

for every $i \in S \subset N$; moreover, as $\rho \rightarrow 1$ we also have

$$
u^{i}\left(\zeta_{(S, f}^{S}(S), k\right)=\operatorname{Sh}^{i}\left(S, v_{G, \sigma}\right)
$$

for every $i, k \in S \subset N$.

Proof. Similar to the proof of Proposition 4; see in particular the explicit computational formula there. Note that the fixed threats imply that what a coalition can obtain is well defined, in the sense of not depending on the order in which the inactive players have dropped out.

Proposition 5 does show that in a very natural sense the solution concept we develop in this paper, the SP equilibrium of the PC procedure, is an extension to a larger context of the Shapley value solution for TU coalitional form games.

What happens in the general NTU case? One may conjecture, as in Hart and Mas-Colell (1996a), that as $\rho$ approaches 1 the SP equilibrium payoffs approach a Maschler-Owen (1992) consistent NTU value of $\left(N, V_{G, \sigma}\right)$. It is not difficult to see that this is indeed the case if, for every $S$, the limit of the SP equilibrium payoffs

7. That is, for every $S \subset N$ there is a real number $v(S)$ such that $c \in V_{G, \sigma}(S)$ and $c^{i} \geq r^{i}$ for all $i \in S$ if and only if $\sum_{i \in S} c^{i} \leq v(S)$. 
of $S$ lies in a smooth piece of the efficient boundary of ${ }^{8} V_{G, \sigma}(S)$. Because this set is a convex polytope, the condition amounts to the requirement that each limit lie in the interior of some $(|S|-1)$-dimensional face of the polytope. In particular, this will be automatically satisfied if $\left(N, V_{G, \sigma}\right)$ is a hyperplane game (see Maschler and Owen (1989); of course, only the individually rational region matters, as in Proposition 5 for the TU case). But a general analysis of the non-smooth case is needed.

\section{Games with Damaging Actions}

Are there classes of games in strategic form that, from the standpoint of the PC procedure, lend themselves to being summarized by means of the coalitional form of cooperative game theory? Presumably, these would be concrete specifications of the c-games of Shapley-Shubik.

In this section we exhibit one such class of games by presenting a property of strategic forms that, for TU games, implies the existence of an SP equilibrium enjoying the fixed-threat property. The NTU case is discussed in the next section.

It is reasonable to expect that the strategic linkage through threats is bound to be simpler in situations where there is some form of "strategic dominance" or "universality" in the threats used by players. This suggests the following.

DEFINITION 2. Given a game $G$, a player $k \in N$ has a damaging action $d^{k} \in A^{k}$ if $u^{i}\left(d^{k}, a^{N \backslash k}\right) \leq u^{i}(a)$ for every action profile $a \in A$ and every player $i \neq k$. A game $G$ is a $d$-game if every player $k \in N$ has a damaging action.

That is, a d-game is such that whatever the play is, if player $k$ switches his action to $d^{k}$ then the payoffs of all the other players decrease or stay the same; being a d-game is thus a strong requirement. The next proposition shows that, indeed, the "d" concept helps relate our approach to cooperative game theory.

Proposition 6. Let $G$ be a strategic TU game. Suppose that $G$ is a d-game. Then there exists a fixed-threat SP equilibrium of the PC procedure where each player $i$ uses a damaging action as threat.

Proof. Let $d^{k}$ be a damaging action of player $k$. Recall (Proposition 4) that at an SP equilibrium of a strategic TU game a proposer $k$ chooses a threat $x^{k} \in \Delta\left(A^{k}\right)$ at state $\omega=\left(S, b^{N \backslash S}\right)$ so as to minimize $v\left(S \backslash k,\left(b^{N \backslash S}, x^{k}\right)\right)$, the sum of the payoffs of the remaining players if the proposer becomes inactive. Obviously, the pure threat $d^{k}$ will do the job for $k$, at any state.

8. See Hart and Mas-Colell (1996a), Proof of Proposition 8 (with the correction at 〈http://www.ma.huji.ac.il/hart/abs/nbarg.html $\rangle$ ), for the reason for the smoothness requirement. 


\section{Market Games Are Not "c-Games"}

Propositions 5 and 6 highlight in a clear way the relationship between the bargaining theory we develop in this paper and classical cooperative game theory: If threats are "self-evident" then they can be taken as fixed threats, a coalitional form emerges in the obvious manner, and the analysis can proceed by taking the coalitional form as the basic datum and appealing to the extensive and rich theory of cooperative games. But Proposition 6 was stated for the TU case. In this section we shall see by means of an example that the result is no longer true for the general NTU situation and that this is so for entirely non-pathological reasons, that is, for reasons that seem inherent in the nature of strategic bargaining among many players. It is therefore very questionable whether, even under the strong hypothesis of the players having damaging actions, bargaining theory in the strategic form can justifiably be factored through cooperative game theory (except in the TU case).

The example will be built over a pure exchange economy satisfying the standard conditions (no externalities, concavity, and monotonicity of preferences, etc.). We choose this framework because exchange economies have been thought to be the paradigmatic cases of c-games, that is, the sort of games where the selfevident coalitional form was fully adequate (see Shubik 1983, p. 131). We shall see in the example below that the obvious damaging threat of never sharing your endowment is not always the optimal threat! The phenomenon is related to the well-known transfer and endowments paradoxes of general equilibrium theory (see, e.g., Postlewaite (1979); also Mas-Colell (1976)), but we should emphasize that here these emerge internally to the theory, namely, within well-specified rules of a game.

The example is of an exchange economy with 4 commodities and 3 traders. Let the commodities be $b, c, f, g$, and the traders, 1, 2, 3. The initial endowments are

$$
\begin{aligned}
e^{1} & =(0,0,1,1), \\
e^{2} & =(0,1,0,0), \\
e^{3} & =(1,0,0,0),
\end{aligned}
$$

and the utility functions are

$$
\begin{aligned}
& u^{1}(b, c, f, g)=b \\
& u^{2}(b, c, f, g)=b+c-1, \\
& u^{3}(b, c, f, g)=\frac{1}{2} c+\max _{b^{\prime}+b^{\prime \prime}=b} b^{\prime}, b^{\prime \prime} \geq 0\left\{\frac{1}{2} \min \left\{b^{\prime}, f\right\}+\min \left\{b^{\prime \prime}, g\right\}\right\} .
\end{aligned}
$$


The goods $b$ and $c$ are mediums of exchange ("money"); player 3 has a "technology" that takes $b$ as input and transforms it into "utils" subject to capacity constraints determined by $f$ and $g$, where the productivity through $g$ is twice as high as the one through $f$.

We make the exchange economy into a strategic game in a natural way, as first formally suggested by Scarf (1971): Each player $i$ distributes his endowment $e^{i}$ among the 3 players: $e^{i}=\sum_{j=1}^{3} d^{i, j}$, where $d^{i, j} \in \mathbb{R}_{+}^{4}$ is the bundle transferred from $i$ to $j$; the outcome (final holding) of player $j$ is thus $h^{j}=\sum_{i=1}^{3} d^{i, j}$, and his payoff is $w^{j}=u^{j}\left(h^{j}\right)$.

Note that in this game every player has a damaging action: to keep all his endowment for himself (i.e., $d^{i, i}=e^{i}$ ). Suppose first that these are indeed the threats; the resulting coalitional function (in the individually rational region, i.e., where all payoffs are nonnegative) is a TU game for all coalitions except $\{2,3\}$. We get $v(i)=0$ for all $i, v(1,2)=0$, and $v(1,3)=v(1,2,3)=1$. As for $\{2,3\}$, the Pareto efficient boundary lies on the line $w^{2}+2 w^{3}=1$. Computing the payoff vectors yields $^{9}(0,0, \cdot)$ for $\{1,2\},(1 / 2, \cdot, 1 / 2)$ for $\{1,3\}$, and $(\cdot, 1 / 2,1 / 4)$ for $\{2,3\}$; extending them to efficient payoff vectors for the grand coalition $N=$ $\{1,2,3\}$ and then averaging gives the final outcome of ${ }^{10}(1 / 4,1 / 6,7 / 12)$.

However, this does not yield an equilibrium, because player 1 has a better threat when he is the proposer in the grand coalition, namely, to transfer his unit of the $f$ good to player 3 . Notice that this threat does not change the nonnegative attainable set for coalition $\{2,3\}$, but player 3 now gets by himself $1 / 2$ rather than 0; see Figure 1. The negotiating terms in coalition $\{2,3\}$ have been altered, and the outcome of this coalition becomes $(\cdot, 0,1 / 2)$; this implies that player 1 can make a demand of $1 / 2$ (instead of the $1 / 4$ that he could ask for when the threat was to keep his own resources). The outcome of the grand coalition is now $(1 / 3,0,2 / 3)$-and so player 1's payoff has increased from $1 / 4$ to $1 / 3$ by the above deviation. In a sense, by this action player 1 has successfully manipulated in his favor the bargaining between 2 and 3 (note that this could not happen in the TU case, because only the total payoff of $\{2,3\}$ matters to 1 ).

So, what are the SP equilibria in this example? Because the efficient boundaries of the attainable sets for $\{1,2,3\}$ and all coalitions except $\{2,3\}$ are TU, keeping one's endowment is optimal, except for player 1 in the grand coalition. His threat in this case must minimize the sum of the payoffs of 2 and 3 in the subgame after 1 becomes inactive (since 1 gets the difference between $v(1,2,3)=1$ and that sum). Now this sum is at least $1 / 2$, because the outcome $(\cdot, 0,1 / 2)$ is always feasible for $\{2,3\}$ (even without any transfers). By transferring 1 unit of good $f$ to player 3 , player 1 makes sure that $(\cdot, 0,1 / 2)$ is necessarily the outcome of $\{2,3\}-$ so this is the optimal threat of player 1 in coalition $\{1,2,3\}$. Thus

9. A dot (.) is used for the coordinate of the missing player.

10. See Hart (2004, Section 5) for a similar computation. 


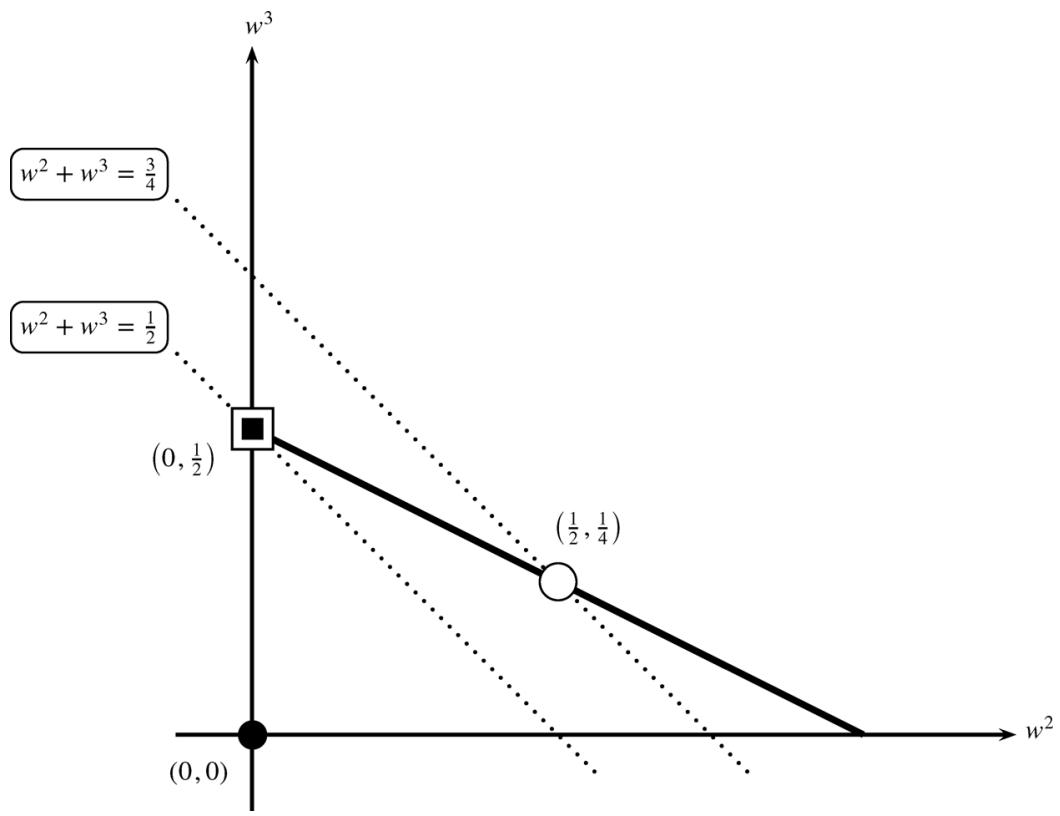

Figure 1. The outcomes of the singletons $\{2\}$ and $\{3\}$ (small black circle), and the outcome of coalition $\{2,3\}$ (large white circle) for the damaging threats; the same outcomes for the optimal threats (small black square and large white square).

the unique SP equilibrium payoffs for the grand coalition are $(1 / 3,0,2 / 3)$, as seen above. Note in particular that the unique optimal threat of 1 in $\{1,3\}$ is to keep his own endowment, and so the SP equilibria ${ }^{11}$ do not have the fixed-threat property. $^{12}$

In conclusion, strategic market games, a classical instance of the so-called cgames, are not really c-games: One cannot simply define the coalitional function as what a coalition can do with the total endowment of its members (except in the TU case). Our point, however, is more general. In the above example player 1 , by using a suitable threat (which is not a damaging threat), can alter - to his advantage - the relative bargaining powers of players 2 and 3 in the subsequent negotiation. In the general NTU case, where the specific subsequent agreement of a coalition of players matters to the proposer (whereas in the TU case only the sum of payoffs matters to him), this phenomenon is bound to be pervasive.

11. Although the SP equilibrium outcomes are unique, the strategies are not (for example, player 1 's threat in coalition $\{1,2\}$ is arbitrary).

12. The curious reader may wonder what the Walrasian equilibrium payoffs are in this example. They are $(0,0,1)$, which arise from zero prices for goods $f$ and $g$, and a positive price for $b$ that is no more than twice the price for $c$. 
From a different perspective, we can view the foregoing analysis as underlying the existence of a substantial theoretical gap between the TU and the NTU situations. One cannot take for granted that the interesting phenomena that may hold for the former will carry over to the latter (for a different question-the equivalence principle — we made a similar point in Hart and Mas-Colell 1996b).

Remark 5. We follow tradition and do not define precisely the concept of a c-game; as Shubik (1983, p. 131) says: "We do not attempt a categorical definition of this term. What is adequate in a given instance may well depend on the solution concept that we intend to employ. To say that a game is a c-game is merely a way of asserting that nothing essential to the ultimate purpose of the model is lost in the process of condensing the extensive or strategic description into a characteristic function." Myerson (1984, p. 88) gives a precise definition of "games with orthogonal coalitions," a significant class of c-games that includes exchange economies (without externalities); however, from our point of view, it has the drawback that it is not tied to a strategic form game (rather, it relies on a setup where each coalition has its own action set ${ }^{13}$ ). How could we attempt a definition of a c-game within the strategic-form-cum-bargaining-procedure approach taken in this paper? Because, at a minimum, there should be an obvious method to construct a natural coalitional form, we could require as part of the definition the existence of a damaging action for each player (one could of course consider other possibilities to singularize an action to be played by a player outside a coalition). In addition, it should also be the case that for the given bargaining procedure (or, for all procedures in some reasonable class), only the coalitional form matters; more precisely, the damaging actions are the fixed threats of an SP equilibrium of the bargaining procedure (and therefore any two c-games with the same "canonical" coalitional form will lead to the same solution). What we have shown is that for such a definition, exchange economies (viewed as strategic form games à la Scarf 1971) are c-games in the TU case, but not necessarily in the NTU case.

\section{Summary and Extensions}

In this paper we have have provided a bargaining procedure that operates directly on the strategic form of a game. It is inspired by Hart and Mas-Colell (1996a) and Nash (1953), and has as an essential feature the use and commitment of players to threats. We have studied its basic properties, as well as the relationships with the approaches directly operating on the coalitional form of a game. We have seen that

13. In particular, in the pure exchange case, the set of actions of a coalition coincides with all possible transfers of goods among its members. 
the connection can be easily made in the TU case, in the sense that for the natural special case (of strategic games with damaging actions) our procedure could be viewed as operating directly on a coalitional form. Yet, this is not true for the general NTU case, even for situations such as exchange economies, traditionally thought of as very well behaved in this respect.

We view this paper as a first step in a general research program that analyzes cooperation based on the strategic form. There are many issues in need of further clarification and study, and there are many avenues for extensions. We mention some:

1. Relax the commitment-to-threats feature; not to the extent of making it impossible (the theory would then change substantially; see Houba and Bolt 2002), but perhaps by simply making it an option available to players.

2. Consider variations of the PC procedure:

a. A threat $x^{k} \in \Delta\left(A^{k}\right)$ is not realized immediately after a rejected proposer $k$ becomes inactive, but rather at the end of the procedure. Thus a state consists of the set of active players $S$ together with the fixed mixed actions of the inactive players $\left(x^{i}\right)_{i \in N \backslash S} \in \prod_{i \in N \backslash S} \Delta\left(A^{i}\right)$.

b. Dispense with the threats altogether and make the inactive players lose their power to choose their actions; thus a proposal is now a $z^{N} \in \Delta\left(A^{N}\right)$, but only the active players are asked to accept it.

3. Propose and study bargaining procedures that correspond to the Harsanyi $(1959,1963) \mathrm{N}$-person generalization of the two-person variable-threat game of Nash (1953).

4. Propose and study bargaining procedures, for instance in the spirit of Perry and Reny (1994), that lead to core-like solutions rather than value-like solutions (as ours do). Moreover, try to identify general classes of procedures that include these as special cases, and understand what drives the different results.

5. Characterize situations where damaging actions and fixed threats obtain. This may be interesting, in particular, in the context of the exchange economies of Section 7: under which conditions (beyond being TU) will the damaging actions (i.e., retaining the endowments) emerge as fixed threats at an SP equilibrium?

6. In terms of cooperative game theory, the SP equilibria of the PC procedure define a solution concept on the class of strategic games. It is natural to look for axiomatic characterizations of this solution (see Hart (2005) for an axiomatization of the NTU-value obtained from the Hart and Mas-Colell (1996a) procedure).

7. When combined with known results-in particular, the equivalence theorems for the Shapley value (see Hart (2002) for a survey) - the conclusions of Sections 5 and 6 for TU exchange economies have some striking 
consequences. If, in a precise but not particularly restrictive sense, the number of players $N$ is large, then the SP equilibria of the PC procedure (for the natural strategic form considered in Section 7) will be nearly Walrasian; that is to say, it is as if the proposer were to set up a price system at which all the traders would trade. This raises the following question: What can one say when $N$ is large in the general NTU case? In particular, would the convergence to Walrasian equilibria still obtain in the subclass of economies where the SP equilibrium has the fixed-threat property (of course, the fixed threat will then be the damaging action of retaining the endowments)?

8. Computational matters are relevant and may be well worth studying. There is a sense in which the computation of an SP equilibrium of the PC procedure should be easier in the TU case. To take the simplest case consider $\rho=$ 0 , which eliminates the need to compute fixed points. In the TU case, the determination of the optimal threat of a player $i$ at the starting state $(N, \cdot)$ depends only on the aggregate utilities that can be obtained by the coalitions $N$ and $N \backslash\{i\}$, and one does not need to know the equilibrium payoffs and the optimal threats for $N \backslash\{i\}$, nor for any smaller subcoalitions. Consequently, the number of optimal threats that need to be considered in the computation of an SP equilibrium depends only on the cardinality of $N$ and not on that of the action sets. In the general NTU case, however, in order to determine the optimal threat of $i$ in the starting state $(N, \cdot)$ one needs to have already solved the optimal threats' problems for all subsequent states, which is done starting with singleton coalitions and going up in size. The number of such problems is given by the number of states, and thus depends not only on the cardinality of $N$, but also on the cardinality of the action sets.

9. In general, there may be multiple SP equilibria; this multiplicity is due, first, to the fixed-point conditions (4), and second, to the fact that the computation starts from singleton coalitions and moves up, and thus each time that there is more than one optimal threat one gets different "branches" of equilibria. It would be interesting to find out relevant classes of games where the SP equilibria, or the SP equilibrium payoffs, are unique (cf. Proposition 5 in the TU case).

10. In the example of Section 7, identify the class of bargaining procedures for which the damaging threat is not always optimal (we suspect that this phenomenon is quite robust, and not limited to the specific PC procedure).

\section{References}

Aumann, Robert J. (1959). “Acceptable Points in General Cooperative $n$-Person Games.” In Contributions to the Theory of Games IV, Annals of Mathematics Study 40, edited by A. W. Tucker and R. D. Luce. Princeton University Press. 
Binmore, Ken, Martin J. Osborne, and Ariel Rubinstein (1992). "Noncooperative Models of Bargaining." In Handbook of Game Theory, with Economic Applications, Volume 1, Chapter 7, edited by R. J. Aumann and S. Hart. North-Holland.

de Clippel, Geoffroy, and Roberto Serrano (2008). "Marginal Contributions and Externalities in the Value.” Econometrica, 76, 1413-1436.

Harsanyi, John C. (1959). "A Bargaining Model for the Cooperative $n$-Person Game.” In Contributions to the Theory of Games IV, Annals of Mathematics Study 40, edited by A. W. Tucker and R. D. Luce. Princeton University Press.

Harsanyi, John C. (1963). "A Simplified Bargaining Model for the $n$-Person Cooperative Game.” International Economic Review, 4, 194-220.

Hart, Sergiu (2002). "Values of Perfectly Competitive Economies." In Handbook of Game Theory, with Economic Applications, Volume 3, Chapter 57, edited by R. J. Aumann and S. Hart. North-Holland.

Hart, Sergiu (2004). "A Comparison of Non-Transferable Utility Values.” Theory and Decision, $56,35-46$.

Hart, Sergiu (2005). "An Axiomatization of the Consistent Non-Transferable Utility Value." International Journal of Game Theory, 33, 355-366.

Hart, Sergiu, and Andreu Mas-Colell (1996a). "Bargaining and Value." Econometrica, 64, 357-380.

Hart, Sergiu, and Andreu Mas-Colell (1996b). "Harsanyi Values of Large Economies: NonEquivalence to Competitive Equilibria." Games and Economic Behavior, 13, 74-99.

Hart, Sergiu, and Andreu Mas-Colell, editors (1997). Cooperation: Game-Theoretic Approaches, NATO ASI, Series F, Volume 155. Springer.

Hildenbrand, Werner (1974). Core and Equilibria of a Large Economy. Princeton University Press.

Houba, Harold, and Wilko Bolt (2002). Credible Threats in Negotiations: A Game-Theoretic Approach. Kluwer.

Macho-Stadler, Inés, David Pérez-Castrillo, and David Wettstein (2007). "Sharing the Surplus: An Extension of the Shapley Value for Environments with Externalities." Journal of Economic Theory, 135, 339-356.

Mas-Colell, Andreu (1976). "En Torno a una Propriedad Poco Atractiva del Equilibrio Competitivo." Moneda y Credito, 136, 11-27.

Maschler, Michael, and Guillermo Owen (1989). "The Consistent Shapley Value for Hyperplane Games." International Journal of Game Theory, 18, 389-407.

Maschler, Michael, and Guillermo Owen (1992). "The Consistent Shapley Value for Games without Side Payments." In Rational Interaction, edited by R. Selten. Springer.

Maskin, Eric (2003). "Bargaining, Coalitions and Externalities." Presidential Address to the Econometric Society. Institute for Advanced Study, Princeton (Working paper).

Myerson, Roger B. (1977). "Value of Games in Partition Function Form." International Journal of Game Theory, 6, 23-31.

Myerson, Roger B. (1984). “Cooperative Games with Incomplete Information.” International Journal of Game Theory, 13, 69-96.

Nash, John F. (1950). “The Bargaining Problem.” Econometrica, 18, 155-162.

Nash, John F. (1953). “Two-Person Cooperative Games.” Econometrica, 21, 128-140.

Osborne, Martin J., and Ariel Rubinstein (1990). Bargaining and Markets. Academic Press.

Osborne, Martin J., and Ariel Rubinstein (1994). A Course on Game Theory. MIT Press.

Perry, Motty, and Philip J. Reny (1994). "A Noncooperative View of Coalition Formation and the Core." Econometrica, 62, 795-817.

Postlewaite, Andrew (1979). "Manipulation via Endowments." Review of Economic Studies, 46, 255-262.

Scarf, Herbert E. (1971). "On the Existence of a Cooperative Solution for a General Class of N-Person Games.” Journal of Economic Theory, 3, 169-181. 
Shapley, Lloyd S. (1953). "A Value for $n$-Person Games." In Contributions to the Theory of Games II, Annals of Mathematics Studies 28, edited by H. W. Kuhn and A. W. Tucker. Princeton University Press.

Shapley, Lloyd S. (1969). "Utility Comparison and the Theory of Games." In La Décision, Editions du CNRS.

Shubik, Martin (1983). Game Theory in the Social Sciences. MIT Press.

Thrall, Robert M., and William F. Lucas (1963). " $n$-Person Games in Partition Function Form." Naval Research Logistics Quarterly, 10, 281-298.

von Neumann, John, and Oskar Morgenstern (1944). Theory of Games and Economic Behavior. Princeton University Press. 